\title{
Article
}

\section{New Bounds for the Modified Bessel Function of the First Kind and Toader-Qi Mean}

\section{Ling Zhu}

check for updates

Citation: Zhu, L. New Bounds for the Modified Bessel Function of the First Kind and Toader-Qi Mean. Mathematics 2021, 9, 2867. https:// doi.org/10.3390/math9222867

\section{Academic Editors: Clemente}

Cesarano, Shanhe Wu and Francesco Mainardi

Received: 3 October 2021

Accepted: 9 November 2021

Published: 11 November 2021

Publisher's Note: MDPI stays neutral with regard to jurisdictional claims in published maps and institutional affiliations.

Copyright: (C) 2021 by the author. Licensee MDPI, Basel, Switzerland. This article is an open access article distributed under the terms and conditions of the Creative Commons Attribution (CC BY) license (https:// creativecommons.org/licenses/by/ $4.0 /)$.
Department of Mathematics, Zhejiang Gongshang University, Hangzhou 310018, China; zhuling@zjgsu.edu.cn

Abstract: Let $I_{p}(x)$ be the modified Bessel function of the first kind of order $p$. The upper and lower bounds in the form of simple rational functions about $\cosh t$ and $(\sinh t) / t$ for the function $I_{0}(x)$ are obtained. The corresponding inequalities for the Toader-Qi mean do not match those in the existing literature.

Keywords: bounds; modified Bessel function of the first kind; hyperbolic function; mean; inequality

\section{Introduction}

We know that the modified Bessel function of the first kind of order $p$ is denoted as $I_{p}(x)(([1]$ p. 77), ([2] p. 374)) and has important applications in many fields of natural science. The function $I_{p}(x)$ denotes a class of solutions of the second-order differential equation ([2] p. 374)

$$
x^{2} y^{\prime \prime}(x)+x y^{\prime}(x)-\left(x^{2}+p^{2}\right) y(x)=0,
$$

which can be written as the infinite series

$$
I_{p}(x)=\left(\frac{x}{2}\right)^{p} \sum_{n=0}^{\infty} \frac{(x / 2)^{2 n}}{n ! \Gamma(p+n+1)}, x \in \mathbb{R}, p \in \mathbb{R} \backslash\{-1,-2, \ldots\},
$$

where $\Gamma(x)$ is the gamma function ([2] p. 255). Moreover, the normalized modified Bessel function defined by $\mathcal{I}_{p}: \mathbb{R} \rightarrow[1,+\infty)$ as $\mathcal{I}_{p}(x)=2^{p} \Gamma(p+1) x^{-p} I_{p}(x)$ was considered in [3]. Then, we have

$$
\mathcal{I}_{p+1 / 2}(x)=2^{p+1 / 2} \Gamma(p+3 / 2) x^{-(p+1 / 2)} I_{p+1 / 2}(x) .
$$

Particularly, for $p=0, p=1$ in the above, the function $\mathcal{I}_{p+1 / 2}(x)$ reduces to some elementary functions, as follows ([1] p. 54):

$$
\mathcal{I}_{1 / 2}(x)=\sqrt{\frac{\pi}{2 x}} I_{1 / 2}(x)=\frac{\sinh x}{x}, \mathcal{I}_{3 / 2}(x)=\frac{3}{x} \sqrt{\frac{\pi}{2 x}} I_{3 / 2}(x)=-3\left(\frac{\sinh x}{x^{3}}-\frac{\cosh x}{x^{2}}\right) .
$$

The properties and various inequalities of this special function $\mathcal{I}_{p}(x)$ are widely discussed in References [3-11].

Throughout this article, we assume that $a$ and $b$ are two different positive real numbers. This paper focuses on

$$
I_{0}(x)=\sum_{n=0}^{\infty} \frac{(x / 2)^{2 n}}{n ! \Gamma(n+1)}=\sum_{n=0}^{\infty} \frac{x^{2 n}}{2^{2 n}(n !)^{2}},
$$

which is the modified Bessel function of the first kind of order 0, which is related to the Toader-Qi mean (see [12,13]) defined by

$$
T Q(a, b)=\frac{2}{\pi} \int_{0}^{\pi / 2} a^{\cos ^{2} \theta} b^{\sin ^{2} \theta} d \theta=\sqrt{a b} I_{0}\left(\ln \sqrt{\frac{a}{b}}\right) .
$$


Let

$$
\begin{array}{ll}
A \equiv A(a, b)=\frac{a+b}{2}, & G \equiv G(a, b)=\sqrt{a b}, \\
L \equiv L(a, b)=\frac{a-b}{\ln a-\ln b}, \quad I \equiv I(a, b)=e^{-1}\left(\frac{b^{b}}{a^{a}}\right)^{1 /(b-a)}
\end{array}
$$

and

$$
A_{v} \equiv A_{v}(a, b)=\left(\frac{a^{v}+b^{v}}{2}\right)^{1 / v} \text { if } v \neq 0
$$

be the arithmetic mean, geometric mean, logarithmic mean, exponential mean, and power mean of order $v$, respectively. Then, an inequality chain (see [14-16])

$$
G<L<A_{1 / 3}<\frac{A+2 G}{3}<A_{1 / 2}<\frac{2 A+G}{3}<A_{2 / 3}<I<A_{\ln 2}<A_{1}
$$

holds. Alzer [17] also provided the following result:

$$
\sqrt{A G}<\sqrt{L I}<\frac{L+I}{2}<\frac{A+G}{2} .
$$

From now on, let us focus on the Toader-Qi mean of $I_{0}(x)$. In 2017, Qi, Shi, Liu, and Yang [13] proved that the inequalities

$$
L<T Q<\frac{A+G}{2}<\frac{2 A+G}{3}<I
$$

hold Yang and Chu ([18] Theorem 3.4) established the following results:

$$
\begin{aligned}
L^{3 / 4} A^{1 / 4}<T Q & <\sqrt{L A_{2 / 3}}<\frac{3}{4} L+\frac{1}{4} A, \\
\sqrt{\frac{2 A L}{\pi}} & <T Q<\sqrt{A L}, \\
\sqrt{\left(\frac{2}{\pi} A+1-\frac{2}{\pi}\right) L} & <T Q<\sqrt{\left(\lambda_{0} A+1-\lambda_{0}\right) L},
\end{aligned}
$$

where $\lambda_{0}=0.6766 \ldots$ Yang, Chu, and Song [19] and Yang, Tian, and Zhu ([20] Remark 4) obtained

$$
\sqrt{\frac{e}{\pi}} \sqrt{L I}<\frac{2^{4 / 3}}{\sqrt{\pi}} \sqrt{L A_{2 / 3}}<T Q<\sqrt{L A_{2 / 3}}<\sqrt{L I}
$$

Let $b>a>0$ and $t=\ln \sqrt{a / b}$. Then, those means mentioned above can be represented in terms of hyperbolic functions:

$$
\begin{aligned}
\frac{A}{\bar{G}} & =\cosh t, \frac{L}{G}=\frac{\sinh t}{t}, \frac{I}{G}=\exp \left(\frac{t}{\tanh t}-1\right), \\
\frac{T Q}{G} & =I_{0}(t), \frac{A_{p}}{G}=\cosh ^{1 / p}(p t) \text { for } p \neq 0 .
\end{aligned}
$$

Correspondingly, the inequalities (10)-(14) mentioned above are equivalent to

$$
\begin{gathered}
\frac{\sinh t}{t}<I_{0}(t)<\frac{\cosh t+1}{2}<\frac{2 \cosh t+1}{3}<\exp \left(\frac{t}{\tanh t}-1\right) \\
\left(\frac{\sinh t}{t}\right)^{3 / 4}(\cosh t)^{1 / 4}<I_{0}(t)<\sqrt{\frac{\sinh t}{t} \cosh ^{3 / 2}\left(\frac{2 t}{3}\right)}<\frac{3 \sinh t}{4}+\frac{1}{4} \cosh t
\end{gathered}
$$




$$
\begin{aligned}
\sqrt{\frac{\sinh (2 t)}{\pi t}} & <I_{0}(t)<\sqrt{\frac{\sinh (2 t)}{2 t}} \\
\sqrt{\left(\frac{2}{\pi} \cosh t+1-\frac{2}{\pi}\right) \frac{\sinh t}{t}} & <I_{0}(t)<\sqrt{\left(\lambda_{0} \cosh t+1-\lambda_{0}\right) \frac{\sinh t}{t}}
\end{aligned}
$$

and

$$
\begin{aligned}
& \sqrt{\frac{e}{\pi}} \sqrt{\frac{\sinh t}{t} \exp \left(\frac{t}{\tanh t}-1\right)} \\
< & \frac{2^{3 / 4}}{\sqrt{\pi}} \sqrt{\frac{\sinh t}{t} \cosh ^{3 / 2}\left(\frac{2 t}{3}\right)} \\
< & I_{0}(t)<\sqrt{\frac{\sinh t}{t} \cosh ^{3 / 2}\left(\frac{2 t}{3}\right)}<\sqrt{\frac{\sinh t}{t} \exp \left(\frac{t}{\tanh t}-1\right)}
\end{aligned}
$$

hold for $t>0$.

In this paper, we first consider finding the bound of $T Q(a, b)$ in the following form:

$$
\frac{x_{1} A^{2}+y_{1} A L+z_{1} G^{2}}{A+w_{1} G},
$$

which is equivalent to searching for a bound for $T Q(a, b) / G=I_{0}(t)$ in the form of

$$
\begin{aligned}
\frac{x_{1} A^{2}+y_{1} A L+z_{1} G^{2}}{\left(A+w_{1} G\right) G} & =\frac{x_{1} A^{2}+y_{1} A L+z_{1} G^{2}}{A G+w_{1} G^{2}} \\
& =\frac{x_{1}(A / G)^{2}+y_{1}(A / G)(L / G)+z_{1}}{A / G+w_{1}} \\
& =\frac{x_{1}(\cosh t)^{2}+y_{1}(\cosh t)((\sinh t) / t)+z_{1}}{\cosh t+w_{1}} .
\end{aligned}
$$

In this way, we can consider the power series expansion

$$
\begin{aligned}
& \left(\cosh t+w_{1}\right) I_{0}(t)-\left[x_{1}(\cosh t)^{2}+y_{1}(\cosh t)((\sinh t) / t)+z_{1}\right] \\
= & \left(-x_{1}-y_{1}-z_{1}+w_{1}+1\right)-t^{2}\left(x_{1}+\frac{2}{3} y_{1}-\frac{1}{4} w_{1}-\frac{3}{4}\right) \\
& -t^{4}\left(\frac{1}{3} x_{1}+\frac{2}{15} y_{1}-\frac{1}{64} w_{1}-\frac{35}{192}\right)-t^{6}\left(\frac{2}{45} x_{1}+\frac{4}{315} y_{1}-\frac{1}{2304} w_{1}-\frac{77}{3840}\right) \\
& -t^{8}\left(\frac{1}{315} x_{1}+\frac{2}{2835} y_{1}-\frac{1}{147,456} w_{1}-\frac{143}{114,688}\right) \\
& -t^{10}\left(\frac{2}{14,175} x_{1}+\frac{4}{155,925} y_{1}-\frac{1}{14,745,600} w_{1}-\frac{46,189}{928,972,800}\right)+O\left(t^{12}\right) .
\end{aligned}
$$

When letting

$$
\left\{\begin{array}{c}
-x_{1}-y_{1}-z_{1}+w_{1}+1=0 \\
x_{1}+\frac{2}{3} y_{1}-\frac{1}{4} w_{1}-\frac{3}{4}=0 \\
\frac{1}{3} x_{1}+\frac{2}{15} y_{1}-\frac{1}{64} w_{1}-\frac{35}{192}=0 \\
\frac{2}{45} x_{1}+\frac{4}{315} y_{1}-\frac{1}{2304} w_{1}-\frac{77}{3840}=0
\end{array}\right.
$$


we can obtain that $x_{1}=2263 / 11,456, y_{1}=20,475 / 22,912, z_{1}=1879 / 22,912$ and $w_{1}=31 / 179$ and find that

$$
\begin{aligned}
& \left(\cosh t+\frac{31}{179}\right) I_{0}(t)-\left[\frac{2263}{11,456}(\cosh t)^{2}+\frac{20,475}{22,912} \frac{\sinh t \cosh t}{t}+\frac{1879}{22,912}\right] \\
= & -\frac{209}{21,995,520} t^{8}-\frac{16,217}{15,242,895,360} t^{10}+O\left(t^{12}\right),
\end{aligned}
$$

which motivates us to prove the inequality

$$
\begin{aligned}
I_{0}(t) & <\frac{\frac{2263}{11,456}(\cosh t)^{2}+\frac{20,475}{22,912} \frac{\sinh t \cosh t}{t}+\frac{1879}{22,912}}{\cosh t+\frac{31}{179}} \\
& =\frac{8284 t+20,475 \sinh 2 t+4526 t \cosh 2 t}{256 t(179 \cosh t+31)}, t>0 .
\end{aligned}
$$

Similarly, we can find the bound for $T Q(a, b)$ in the form of

$$
\frac{x_{2} L^{2}+y_{2} L G+z_{2} G^{2}}{A+w_{2} G}
$$

and obtain the exact bound by the approximation method used just now. The main conclusions of this paper are as follows.

Theorem 1. The inequality

$$
\begin{aligned}
I_{0}(t) & <\frac{\frac{2263}{11,456}(\cosh t)^{2}+\frac{20,475}{22,912} \frac{\sinh t \cosh t}{t}+\frac{1879}{22,912}}{\cosh t+\frac{31}{179}} \\
& =\frac{8284 t+20,475 \sinh 2 t+4526 t \cosh 2 t}{256 t(179 \cosh t+31)}
\end{aligned}
$$

holds for $t>0$, or equivalently,

$$
\begin{aligned}
T Q(a, b) & <\frac{\frac{2263}{11,456} A^{2}+\frac{20,475}{22,912} A L+\frac{1879}{22,912} G^{2}}{A+\frac{31}{179} G} \\
& =\frac{20,475 A L+4526 A^{2}+1879 G^{2}}{128(179 A+31 G)}
\end{aligned}
$$

holds for $a, b>0$ with $a \neq b$.

Theorem 2. The inequality

$$
\begin{aligned}
I_{0}(t) & >\frac{\frac{4659}{568}\left(\frac{\sinh t}{t}\right)^{2}+\frac{7941}{284} \frac{\sinh t}{t}-\frac{4877}{568}}{\cosh t+\frac{1887}{71}} \\
& =\frac{4659 \cosh 2 t+31,764 t \sinh t-9754 t^{2}-4659}{16 t^{2}(71 \cosh t+1887)}
\end{aligned}
$$

holds for $t>0$, or equivalently,

$$
\begin{aligned}
T Q(a, b) & >\frac{\frac{4659}{568} L^{2}+\frac{7941}{284} L G-\frac{4877}{568} G^{2}}{A+\frac{1887}{71} G} \\
& =\frac{4659 L^{2}+15,882 G L-4877 G^{2}}{8(71 A+1887 G)}
\end{aligned}
$$

holds for $a, b>0$ with $a \neq b$. 


\section{Lemmas}

The precise power series representation of $(\cosh t) I_{0}(t)$ is required to establish two theorems in this article. We thank Prof. Dr. H. W. Gould for compiling an important result on the finite sum of combinatorial numbers in his monograph [21], which we consider as the substance of Lemma 1.

Lemma 1 ([21] (3.175)). Let $\left(\begin{array}{l}n \\ k\end{array}\right)$ be the number of different ways to choose $k$ elements from a given set with $n$ distinct elements, that is

$$
\left(\begin{array}{l}
n \\
k
\end{array}\right)=\frac{n !}{k !(n-k) !}
$$

Then, for all $x \in \mathbb{R}$,

$$
\sum_{k=0}^{n}\left(\begin{array}{c}
x \\
2 k
\end{array}\right)\left(\begin{array}{c}
x-2 k \\
n-k
\end{array}\right) 2^{2 k}=\left(\begin{array}{c}
2 x \\
2 n
\end{array}\right)
$$

Lemma 2. We have

$$
I_{0}(t) \cosh t=\sum_{n=0}^{\infty} \frac{(4 n-1) ! !}{[(2 n) !]^{2}} t^{2 n}=: \sum_{n=0}^{\infty} \kappa_{n} t^{2 n}
$$

where

$$
\kappa_{n}=\frac{(4 n-1) ! !}{[(2 n) !]^{2}} .
$$

Proof. Using the Cauchy product formula, we have

$$
\begin{aligned}
I_{0}(t) \cosh t & =\left(\sum_{n=0}^{\infty} \frac{1}{2^{2 n}(n !)^{2}} t^{2 n}\right)\left(\sum_{n=0}^{\infty} \frac{1}{(2 n) !} t^{2 n}\right) \\
& =\sum_{n=0}^{\infty} \sum_{k=0}^{n} \frac{1}{2^{2 k}(k !)^{2}} \frac{1}{(2 n-2 k) !} t^{2 n} \\
& =: \sum_{n=0}^{\infty} \kappa_{n} t^{2 n},
\end{aligned}
$$

where

$$
\kappa_{n}=\sum_{k=0}^{n} \frac{1}{2^{2 k}(k !)^{2}} \frac{1}{(2 n-2 k) !}
$$

Since

$$
\begin{aligned}
\left(\begin{array}{c}
2 n \\
2 k
\end{array}\right) & =\frac{(2 n) !}{(2 k) !(2 n-2 k) !} \\
(2 k) ! & =(k !)^{2}\left(\begin{array}{c}
2 k \\
k
\end{array}\right)
\end{aligned}
$$

we have

$$
\begin{aligned}
\kappa_{n} & =\sum_{k=0}^{n} \frac{1}{2^{2 k}(k !)^{2}(2 n-2 k) !}=\frac{1}{(2 n) !} \sum_{k=0}^{n} \frac{(2 k) !}{2^{2 k}(k !)^{2}}\left(\begin{array}{c}
2 n \\
2 k
\end{array}\right) \\
& =\frac{1}{(2 n) !} \sum_{k=0}^{n} \frac{(k !)^{2}\left(\begin{array}{c}
2 k \\
k
\end{array}\right)}{2^{2 k}(k !)^{2}}\left(\begin{array}{l}
2 n \\
2 k
\end{array}\right)=\frac{1}{(2 n) !} \sum_{k=0}^{n} \frac{1}{2^{2 k}}\left(\begin{array}{c}
2 n \\
2 k
\end{array}\right)\left(\begin{array}{c}
2 k \\
k
\end{array}\right) .
\end{aligned}
$$


Letting $x=2 n$ in (24) gives

$$
\sum_{k=0}^{n}\left(\begin{array}{c}
2 n \\
2 k
\end{array}\right)\left(\begin{array}{c}
2 n-2 k \\
n-k
\end{array}\right) 2^{2 k}=\left(\begin{array}{c}
4 n \\
2 n
\end{array}\right) .
$$

On the one hand, let $n-k=l$. Then

$$
\begin{aligned}
\sum_{k=0}^{n}\left(\begin{array}{c}
2 n \\
2 k
\end{array}\right)\left(\begin{array}{c}
2 n-2 k \\
n-k
\end{array}\right) 2^{2 k} & =\sum_{l=0}^{n}\left(\begin{array}{c}
2 n \\
2 n-2 l
\end{array}\right)\left(\begin{array}{c}
2 l \\
l
\end{array}\right) 2^{2(n-l)} \\
& =2^{2 n} \sum_{l=0}^{n} \frac{1}{2^{2 l}}\left(\begin{array}{c}
2 n \\
2 n-2 l
\end{array}\right)\left(\begin{array}{c}
2 l \\
l
\end{array}\right) \\
& =2^{2 n} \sum_{k=0}^{n} \frac{1}{2^{2 k}}\left(\begin{array}{c}
2 n \\
2 n-2 k
\end{array}\right)\left(\begin{array}{c}
2 k \\
k
\end{array}\right) \\
& =2^{2 n} \sum_{k=0}^{n} \frac{1}{2^{2 k}}\left(\begin{array}{c}
2 n \\
2 k
\end{array}\right)\left(\begin{array}{c}
2 k \\
k
\end{array}\right) .
\end{aligned}
$$

On the other hand, we have

$$
\left(\begin{array}{l}
4 n \\
2 n
\end{array}\right)=\frac{(4 n) !}{[(2 n) !]^{2}}
$$

Therefore,

$$
2^{2 n} \sum_{k=0}^{n} \frac{1}{2^{2 k}}\left(\begin{array}{c}
2 n \\
2 k
\end{array}\right)\left(\begin{array}{c}
2 k \\
k
\end{array}\right)=\frac{(4 n) !}{[(2 n) !]^{2}} \Longleftrightarrow \sum_{k=0}^{n} \frac{1}{2^{2 k}}\left(\begin{array}{c}
2 n \\
2 k
\end{array}\right)\left(\begin{array}{c}
2 k \\
k
\end{array}\right)=\frac{(4 n) !}{2^{2 n}[(2 n) !]^{2}}
$$

and

$$
\begin{aligned}
\kappa_{n} & =\frac{1}{(2 n) !} \frac{(4 n) !}{2^{2 n}[(2 n) !]^{2}}=\frac{1}{(2 n) !} \frac{(4 n)(4 n-1)(4 n-2)(4 n-3) \cdots 4 \cdot 3 \cdot 2 \cdot 1}{2^{2 n}[(2 n) !]^{2}} \\
& =\frac{1}{(2 n) !} \frac{(4 n)(4 n-2) \cdots 4 \cdot 2(4 n-1) ! !}{2^{2 n}[(2 n) !]^{2}}=\frac{1}{(2 n) !} \frac{2^{2 n}(2 n) !(4 n-1) ! !}{2^{2 n}[(2 n) !]^{2}} \\
& =\frac{(4 n-1) ! !}{[(2 n) !]^{2}} .
\end{aligned}
$$

\section{Proofs of Theorems 1 and 2}

3.1. Proof of Theorem 1

The inequality to be proved is

$$
\begin{aligned}
f_{1}(t) & =: 8284+20,475 \frac{1}{t} \sinh 2 t+4526 \cosh 2 t \\
& >256(179 \cosh t+31) I_{0}(t)=: g_{1}(t), t>0
\end{aligned}
$$



we have

Using the power series expansion of hyperbolic sine and hyperbolic cosine functions,

$$
\begin{aligned}
f_{1}(t)= & 8284+20,475 \sum_{n=0}^{\infty} \frac{2^{2 n+1}}{(2 n+1) !} t^{2 n}+4526 \sum_{n=0}^{\infty} \frac{2^{2 n}}{(2 n) !} t^{2 n} \\
= & 8284+\sum_{n=0}^{\infty}\left[\frac{20,475 \cdot 2^{2 n+1}}{(2 n+1) !}+\frac{4526 \cdot 2^{2 n}}{(2 n) !}\right] t^{2 n} \\
= & 53,760+36,352 t^{2}+\frac{25,432}{3} t^{4}+\frac{41,504}{45} t^{6} \\
& +\sum_{n=4}^{\infty}\left[\frac{20,475 \cdot 2^{2 n+1}}{(2 n+1) !}+\frac{4526 \cdot 2^{2 n}}{(2 n) !}\right] t^{2 n}
\end{aligned}
$$

Then, using Formulas (5) and (25), we obtain

$$
\begin{aligned}
g_{1}(t)= & 45,824 I_{0}(t) \cosh t+7936 I_{0}(t) \\
= & 45,824 \sum_{n=0}^{\infty} \kappa_{n} t^{2 n}+7936 \sum_{n=0}^{\infty} \frac{1}{2^{2 n}(n !)^{2}} t^{2 n} \\
= & \sum_{n=0}^{\infty}\left[45,824 \kappa_{n}+\frac{7936}{2^{2 n}(n !)^{2}}\right] t^{2 n} \\
= & 53,760+36,352 t^{2}+\frac{25,432}{3} t^{4}+\frac{41,504}{45} t^{6} \\
& +\sum_{n=4}^{\infty}\left[45,824 \kappa_{n}+\frac{7936}{2^{2 n}(n !)^{2}}\right] t^{2 n} .
\end{aligned}
$$

The inequality (27) is proved when the following inequality holds:

$$
\begin{gathered}
45,824 \kappa_{n}+\frac{7936}{2^{2 n}(n !)^{2}}<\frac{20,475 \cdot 2^{2 n+1}}{(2 n+1) !}+\frac{4526 \cdot 2^{2 n}}{(2 n) !}, n \geq 4 \\
\Longleftrightarrow 45,824 \frac{(4 n-1) ! !}{((2 n) !)^{2}}+\frac{7936}{2^{2 n}(n !)^{2}}<\frac{20,475 \cdot 2^{2 n+1}}{(2 n+1) !}+\frac{4526 \cdot 2^{2 n}}{(2 n) !}, n \geq 4 \\
\Longleftrightarrow 45,824 \frac{(4 n-1) ! !}{((2 n) !)^{2}}<\frac{20,475 \cdot 2^{2 n+1}}{(2 n+1) !}+\frac{4526 \cdot 2^{2 n}}{(2 n) !}-\frac{7936}{2^{2 n}(n !)^{2}}, n \geq 4
\end{gathered}
$$

Next, we prove (28) by mathematical induction. It is not difficult to verify that the above Formula (28) is true for $n=4$. Suppose (28) holds for $m$, that is

$$
45,824 \frac{(4 m-1) ! !}{((2 m) !)^{2}}<\frac{20,475 \cdot 2^{2 m+1}}{(2 m+1) !}+\frac{4526 \cdot 2^{2 m}}{(2 m) !}-\frac{7936}{2^{2 m}(m !)^{2}}, m \geq 4 .
$$

By (29) we have

$$
\begin{aligned}
& 45,824 \frac{(4 m+3) ! !}{[(2 m+2) !]^{2}} \\
= & \frac{(4 m+3)(4 m+1)}{(2 m+2)^{2}(2 m+1)^{2}}\left[45,824 \frac{(4 m-1) ! !}{((2 m) !)^{2}}\right] \\
< & \frac{(4 m+3)(4 m+1)}{(2 m+2)^{2}(2 m+1)^{2}}\left[\frac{20,475 \cdot 2^{2 m+1}}{(2 m+1) !}+\frac{4526 \cdot 2^{2 m}}{(2 m) !}-\frac{7936}{2^{2 m}(m !)^{2}}\right] .
\end{aligned}
$$


The inequality (28) is proved when proving

$$
\begin{aligned}
& \frac{(4 m+3)(4 m+1)}{(2 m+2)^{2}(2 m+1)^{2}}\left[\frac{20,475 \cdot 2^{2 m+1}}{(2 m+1) !}+\frac{4526 \cdot 2^{2 m}}{(2 m) !}-\frac{7936}{2^{2 m}(m !)^{2}}\right] \\
< & \frac{20,475 \cdot 2^{2 m+3}}{(2 m+3) !}+\frac{4526 \cdot 2^{2 m+2}}{(2 m+2) !}-\frac{7936}{2^{2 m+2}((m+1) !)^{2}}
\end{aligned}
$$

that is

$$
\begin{aligned}
& \frac{(4 m+3)(4 m+1)}{(2 m+2)^{2}(2 m+1)^{2}} \frac{20,475 \cdot 2^{2 m+1}}{(2 m+1) !}+\frac{(4 m+3)(4 m+1)}{(2 m+2)^{2}(2 m+1)^{2}} \frac{4526 \cdot 2^{2 m}}{(2 m) !} \\
& -\left(\frac{20,475 \cdot 2^{2 m+3}}{(2 m+3) !}+\frac{4526 \cdot 2^{2 m+2}}{(2 m+2) !}\right) \\
< & \frac{(4 m+3)(4 m+1)}{(2 m+2)^{2}(2 m+1)^{2}} \frac{7936}{2^{2 m}(m !)^{2}}-\frac{7936}{2^{2 m+2}((m+1) !)^{2}}
\end{aligned}
$$

or

$$
-2 \times 2^{2 m} \frac{-70,909 m-68,754 m^{2}+36,208 m^{3}+6735}{(2 m+3) !(m+1)(2 m+1)^{2}}<\frac{3968\left(6 m+6 m^{2}+1\right)}{(m+1) !^{2} 2^{2 m}(2 m+1)^{2}} .
$$

Since $36,208 m^{3}-68,754 m^{2}-70,909 m+6735>0$ for $m \geq 4$, the above inequality is obviously true.

The proof of the Theorem 1 has been completed.

\subsection{Proof of Theorem 2}

The conclusion to be proved is equivalent to

$$
\begin{aligned}
f_{2}(t) & =:\left(1136 t^{2} \cosh t+30,192 t^{2}\right) I_{0}(t) \\
& >4659 \cosh 2 t+31,764 t \sinh t-9754 t^{2}-4659=: g_{2}(t), t>0
\end{aligned}
$$

Using Formulas (5) and (25), we obtain

$$
\begin{aligned}
f_{2}(t) & =1136 t^{2} I_{0}(t) \cosh t+30,192 t^{2} I_{0}(t) \\
& =1136 \sum_{n=0}^{\infty} \kappa_{n} t^{2 n+2}+30,192 \sum_{n=0}^{\infty} \frac{1}{2^{2 n}(n !)^{2}} t^{2 n+2} \\
& =\sum_{n=0}^{\infty}\left[1136 \kappa_{n}+\frac{30,192}{2^{2 n}(n !)^{2}}\right] t^{2 n+2} \\
& =31,328 t^{2}+8400 t^{4}+\frac{4073}{6} t^{6}+\frac{2153}{60} t^{8}+\sum_{n=4}^{\infty}\left[1136 \kappa_{n}+\frac{30,192}{2^{2 n}(n !)^{2}}\right] t^{2 n+2} .
\end{aligned}
$$


Using the power series expansion of hyperbolic sine and hyperbolic cosine functions, we have

$$
\begin{aligned}
g_{2}(t)= & 4659 \sum_{n=0}^{\infty} \frac{2^{2 n}}{(2 n) !} t^{2 n}+31,764 \sum_{n=0}^{\infty} \frac{1}{(2 n+1) !} t^{2 n+2}-9754 t^{2}-4659 \\
= & 4659 \sum_{n=-1}^{\infty} \frac{2^{2 n+2}}{(2 n+2) !} t^{2 n+2}+31,764 \sum_{n=0}^{\infty} \frac{1}{(2 n+1) !} t^{2 n+2}-9754 t^{2}-4659 \\
= & 31,328 t^{2}+8400 t^{4}+\frac{4073}{6} t^{6}+\frac{2153}{60} t^{8} \\
& +4659 \sum_{n=4}^{\infty} \frac{2^{2 n+2}}{(2 n+2) !} t^{2 n+2}+31,764 \sum_{n=4}^{\infty} \frac{1}{(2 n+1) !} t^{2 n+2} \\
= & 31,328 t^{2}+8400 t^{4}+\frac{4073}{6} t^{6}+\frac{2153}{60} t^{8} \\
& +\sum_{n=4}^{\infty}\left[\frac{4659 \cdot 2^{2 n+2}}{(2 n+2) !}+\frac{31,764}{(2 n+1) !}\right] t^{2 n+2}
\end{aligned}
$$

The inequality (27) is proved when the following inequality

$$
1136 \kappa_{n}+\frac{30,192}{2^{2 n}(n !)^{2}}>\frac{4659 \cdot 2^{2 n+2}}{(2 n+2) !}+\frac{31,764}{(2 n+1) !}
$$

holds for $n \geq 4$, that is,

$$
\frac{1136(4 n-1) ! !}{((2 n) !)^{2}}+\frac{30,192}{2^{2 n}(n !)^{2}}>\frac{4659 \cdot 2^{2 n+2}}{(2 n+2) !}+\frac{31,764}{(2 n+1) !}, n \geq 4
$$

or

$$
\frac{1136(4 n-1) ! !}{((2 n) !)^{2}}>\frac{4659}{(2 n+2) !} 2^{2 n+2}+\frac{31,764}{(2 n+1) !}-\frac{30,192}{2^{2 n}(n !)^{2}}, n \geq 4
$$

It can be verified that the above formula holds when $n=4$. Suppose that Formula (31) holds for $m$, i.e.,

$$
\frac{1136(4 m-1) ! !}{((2 m) !)^{2}}>\frac{4659}{(2 m+2) !} 2^{2 m+2}+\frac{31,764}{(2 m+1) !}-\frac{30,192}{2^{2 m}(m !)^{2}}, m \geq 4 .
$$

Next, we prove that Formula (31) is also true for $m+1$. By (32), we have

$$
\begin{aligned}
& \frac{1136(4 m+3) ! !}{((2 m+2) !)^{2}} \\
= & \frac{(4 m+3)(4 m+1)}{((2 m+2)(2 m+1))^{2}} \frac{1136(4 m-1) ! !}{((2 m) !)^{2}} \\
> & \frac{(4 m+3)(4 m+1)}{((2 m+2)(2 m+1))^{2}}\left[\frac{4659}{(2 m+2) !} 2^{2 m+2}+\frac{31,764}{(2 m+1) !}-\frac{30,192}{2^{2 m}(m !)^{2}}\right] .
\end{aligned}
$$

In this way, we complete the proof of (31) when proving

$$
\begin{aligned}
& \frac{(4 m+3)(4 m+1)}{((2 m+2)(2 m+1))^{2}}\left[\frac{4659}{(2 m+2) !} 2^{2 m+2}+\frac{31,764}{(2 m+1) !}-\frac{30,192}{2^{2 m}(m !)^{2}}\right] \\
> & \frac{4659}{(2 m+4) !} 2^{2 m+4}+\frac{31,764}{(2 m+3) !}-\frac{30,192}{2^{2 m+2}((m+1) !)^{2}}
\end{aligned}
$$




$$
\begin{aligned}
& \Longleftrightarrow \frac{(4 m+3)(4 m+1)}{((2 m+2)(2 m+1))^{2}} \frac{4659}{(2 m+2) !} 2^{2 m+2}+\frac{(4 m+3)(4 m+1)}{((2 m+2)(2 m+1))^{2}} \frac{31,764}{(2 m+1) !} \\
& -\frac{(4 m+3)(4 m+1)}{((2 m+2)(2 m+1))^{2}} \frac{30,192}{2^{2 m}(m !)^{2}} \\
& >\frac{4659}{(2 m+4) !} 2^{2 m+4}+\frac{31,764}{(2 m+3) !}-\frac{30,192}{2^{2 m+2}((m+1) !)^{2}} \\
& \Longleftrightarrow \frac{(4 m+3)(4 m+1)}{((2 m+2)(2 m+1))^{2}} \frac{4659}{(2 m+2) !} 2^{2 m+2}+\frac{(4 m+3)(4 m+1)}{((2 m+2)(2 m+1))^{2}} \frac{31,764}{(2 m+1) !} \\
& -\left(\frac{4659}{(2 m+4) !} 2^{2 m+4}+\frac{31,764}{(2 m+3) !}\right) \\
& >\frac{(4 m+3)(4 m+1)}{((2 m+2)(2 m+1))^{2}} \frac{30,192}{2^{2 m}(m !)^{2}}-\frac{30,192}{2^{2 m+2}((m+1) !)^{2}} \\
& \Longleftrightarrow \quad \frac{6\left[\begin{array}{c}
\left(74,544 m^{3}+170,830 m^{2}+107,157 m+15,530\right) 2^{2 m} \\
+127,056 m^{5}+719,984 m^{4}+1,503,496 m^{3} \\
+1,413,498 m^{2}+577,046 m+74,116
\end{array}\right]}{(2 m+4) !(m+1)^{2}(2 m+1)^{2}} \\
& >\frac{15,096\left(6 m+6 m^{2}+1\right)}{[(m+1) !]^{2} 2^{2 m}(2 m+1)^{2}} \\
& \Longleftrightarrow \frac{\left[\begin{array}{c}
\left(74,544 m^{3}+170,830 m^{2}+107,157 m+15,530\right) 2^{2 m} \\
+127,056 m^{5}+719,984 m^{4}+1,503,496 m^{3} \\
+1,413,498 m^{2}+577,046 m+74,116
\end{array}\right]}{(2 m+4) !} \\
& >\frac{2516\left(6 m+6 m^{2}+1\right)}{(m !)^{2} 2^{2 m}},
\end{aligned}
$$

that is,

$$
\frac{(m !)^{2} 2^{2 m}}{(2 m+4) !}>\frac{2516\left(6 m+6 m^{2}+1\right)}{\left[\begin{array}{c}
\left(74,544 m^{3}+170,830 m^{2}+107,157 m+15,530\right) 2^{2 m} \\
+127,056 m^{5}+719,984 m^{4}+1,503,496 m^{3} \\
+1,413,498 m^{2}+577,046 m+74,116
\end{array}\right]}
$$

holds for $m \geq 4$.

Then, we use mathematical induction to prove (33). It is not difficult to verify that the above Formula (33) is true for $m=4$. Suppose that (33) holds for $m=n$, i.e.,

$$
\begin{aligned}
f(n)= & : \frac{(n !)^{2} 2^{2 n}}{(2 n+4) !} \\
> & \frac{2516\left(6 n+6 n^{2}+1\right)}{\left[\begin{array}{c}
\left(74,544 n^{3}+170,830 n^{2}+107,157 n+15,530\right) 2^{2 n} \\
+127,056 n^{5}+719,984 n^{4}+1,503,496 n^{3} \\
+1,413,498 n^{2}+577,046 n+74,116
\end{array}\right]}=: g(n), n \geq 4 \text {. (34) }
\end{aligned}
$$


By (34), we have

$$
\begin{aligned}
f(n+1)= & \frac{((n+1) !)^{2} 2^{2 n+2}}{(2 n+6) !}=\frac{4(n+1)^{2}}{(2 n+6)(2 n+5)} \frac{n !^{2} 2^{2 n}}{(2 n+4) !} \\
> & \frac{4(n+1)^{2}}{(2 n+6)(2 n+5)} \frac{2516\left(6 n+6 n^{2}+1\right)}{\left[\begin{array}{c}
\left(74,544 n^{3}+170,830 n^{2}+107,157 n+15,530\right) 2^{2 n} \\
+127,056 n^{5}+719,984 n^{4}+1,503,496 n^{3} \\
+1,413,498 n^{2}+577,046 n+74,116
\end{array}\right]},
\end{aligned}
$$

so the proof of (33) is complete when we can prove that

$$
\begin{aligned}
& \frac{A}{B}=: \frac{2516 \cdot 4(n+1)^{2}\left(6 n+6 n^{2}+1\right)}{(2 n+6)(2 n+5)\left[\begin{array}{c}
\left(74,544 n^{3}+170,830 n^{2}+107,157 n+15,530\right) 2^{2 n} \\
+127,056 n^{5}+719,984 n^{4}+1,503,496 n^{3} \\
+1,413,498 n^{2}+577,046 n+74,116
\end{array}\right]} \\
& >g(n+1) \\
& =\frac{2516\left(6(n+1)+6(n+1)^{2}+1\right)}{\left[\begin{array}{c}
\left.\left(74,544(n+1)^{3}+170,830(n+1)^{2}+107,157(n+1)+15,530\right)\right)^{2 n+2} \\
+127,056(n+1)^{5}+719,984(n+1)^{4}+1,503,496(n+1)^{3} \\
+1,413,498(n+1)^{2}+577,046(n+1)+74,116
\end{array}\right]} \\
& =: \frac{C}{D} \text {. }
\end{aligned}
$$

In fact,

$$
\begin{aligned}
& (A D-B C) / 2516 \\
= & 9318 \times 2^{2 n}\left(\begin{array}{c}
349 n+4227 n^{2}+13,036 n^{3}+17,962 n^{4} \\
+12,512 n^{5}+4296 n^{6}+576 n^{7}-18
\end{array}\right) \\
& -10588(n+3)(n+2)(n+1)\left(\begin{array}{c}
1238 n+2702 n^{2}+2496 n^{3} \\
+1008 n^{4}+144 n^{5}+177
\end{array}\right) \\
> & 0
\end{aligned}
$$

holds for all $n \geq 4$. The above formula is obviously equivalent to

$$
2^{2 n}>\frac{\left[\begin{array}{c}
5294(n+1)(n+2)(n+3) \\
\left(1238 n+2702 n^{2}+2496 n^{3}+1008 n^{4}+144 n^{5}+177\right)
\end{array}\right]}{4659\left[\begin{array}{c}
349 n+4227 n^{2}+13,036 n^{3}+17,962 n^{4} \\
+12,512 n^{5}+4296 n^{6}+576 n^{7}-18
\end{array}\right]}, n \geq 4 .
$$

We use mathematical induction for the third time in the process of proving Theorem 2. The Formula (36) is obviously true for $n=4$. We assume that (36) holds for $n=m$, that is,

$$
2^{2 m}>\frac{\left[\begin{array}{c}
5294(m+1)(m+2)(m+3) \\
\left(1238 m+2702 m^{2}+2496 m^{3}+1008 m^{4}+144 m^{5}+177\right)
\end{array}\right]}{4659\left[\begin{array}{c}
349 m+4227 m^{2}+13,036 m^{3}+17,962 m^{4} \\
+12,512 m^{5}+4296 m^{6}+576 m^{7}-18
\end{array}\right]} .
$$


It is proved below that (36) holds for $n=m+1$. By (37), we have

$$
2^{2 m+2}=4 \cdot 2^{2 m}>4 \cdot \frac{\left[\begin{array}{c}
5294(m+1)(m+2)(m+3) \\
\left(1238 m+2702 m^{2}+2496 m^{3}+1008 m^{4}+144 m^{5}+177\right)
\end{array}\right]}{4659\left[\begin{array}{c}
349 m+4227 m^{2}+13,036 m^{3}+17,962 m^{4} \\
+12,512 m^{5}+4296 m^{6}+576 m^{7}-18
\end{array}\right]},
$$

so the proof of (36) is complete when proving

$$
\begin{aligned}
& 4 \cdot \frac{5294}{4659} \frac{\left[\begin{array}{c}
(m+1)(m+2)(m+3) \\
\left(1238 m+2702 m^{2}+2496 m^{3}+1008 m^{4}+144 m^{5}+177\right)
\end{array}\right]}{\left[\begin{array}{c}
349 m+4227 m^{2}+13,036 m^{3}+17,962 m^{4} \\
+12,512 m^{5}+4296 m^{6}+576 m^{7}-18
\end{array}\right]} \\
& >\frac{5294}{4659} \frac{\left[\begin{array}{c}
(m+2)(m+3)(m+4) \\
\left(\begin{array}{c}
1238(m+1)+2702(m+1)^{2}+2496(m+1)^{3} \\
+1008(m+1)^{4}+144(m+1)^{5}+177
\end{array}\right)
\end{array}\right]}{\left[\begin{array}{c}
349(m+1)+4227(m+1)^{2}+13,036(m+1)^{3}+17,962(m+1)^{4} \\
+12,512(m+1)^{5}+4296(m+1)^{6}+576(m+1)^{7}-18
\end{array}\right]} \\
& \Longleftrightarrow \quad \frac{a}{b}=: \frac{4(m+1)\left[\begin{array}{c}
1238 m+2702 m^{2}+2496 m^{3} \\
+1008 m^{4}+144 m^{5}+177
\end{array}\right]}{\left[\begin{array}{c}
349 m+4227 m^{2}+13,036 m^{3}+17,962 m^{4} \\
+12,512 m^{5}+4296 m^{6}+576 m^{7}-18
\end{array}\right]} \\
& >\frac{(m+4)\left[\begin{array}{c}
1238(m+1)+2702(m+1)^{2}+2496(m+1)^{3} \\
+1008(m+1)^{4}+144(m+1)^{5}+177
\end{array}\right]}{\left[\begin{array}{c}
349(m+1)+4227(m+1)^{2}+13,036(m+1)^{3}+17,962(m+1)^{4} \\
+12,512(m+1)^{5}+4296(m+1)^{6}+576(m+1)^{7}-18
\end{array}\right]} \\
& =\quad: \frac{c}{d} \text {. }
\end{aligned}
$$

We calculated that

$$
\begin{aligned}
a d-b c= & 248,832 m^{13}+5,505,408 m^{12}+55,199,232 m^{11}+332,101,728 m^{10} \\
& +1,337,449,104 m^{9}+3,805,389,696 m^{8}+7,862,263,692 m^{7} \\
& +11,923,195,508 m^{6}+13,236,235,612 m^{5}+10,578,592,104 m^{4} \\
& +5,876,921,975 m^{3}+2,125,982,239 m^{2}+440,485,650 m+38,040,600 \\
> & 0 .
\end{aligned}
$$

\section{Comparisons of New and Old Results}

Through observations, in the literature, the more accurate upper and lower bounds of $I_{0}(t)$ are the second inequality in (11)

$$
I_{0}(t)<\sqrt{\frac{\sinh t}{t} \cosh ^{3 / 2}\left(\frac{2 t}{3}\right)}
$$

and the left-hand side of the inequality in (11)

$$
\left(\frac{\sinh t}{t}\right)^{3 / 4}(\cosh t)^{1 / 4}<I_{0}(t)
$$


Next, we compare them with the results of this paper.

(i) The numerical results show that inequality (20) is sharper than the one (38) on $(0,5.2904)$ while that inequality $(38)$ is sharper than the one $(20)$ on $(5.2904, \infty)$. In other words, the two inequalities (21) and the second inequality in (11) cannot be compared.

(ii) The numerical results show that inequality (22) is sharper than the one (39) on $(0,4.916)$ while that inequality (39) is sharper than the one $(22)$ on $(4.916, \infty)$. In other words, the two inequalities (23) and the left-hand side of (11) cannot be compared.

\section{Conclusions} equality

Let $I_{p}(x)$ be the modified Bessel function of the first kind of order $p$. The double in-

$$
\frac{4659 \cosh 2 t+31,764 t \sinh t-9754 t^{2}-4659}{16 t^{2}(71 \cosh t+1887)}<I_{0}(t)<\frac{8284 t+20,475 \sinh 2 t+4526 t \cosh 2 t}{256 t(179 \cosh t+31)}
$$

was proved to hold for $t>0$. The corresponding inequalities for a Toader-Qi mean are not in accordance with the conclusions in previous literature.

Funding: This research was funded by The Natural Science Foundation of China OF FUNDER grant number 61772025 .

Institutional Review Board Statement: Not applicable.

Informed Consent Statement: Not applicable.

Acknowledgments: The author is thankful to the reviewers for their careful corrections to and valuable comments on the original version of this paper.

Conflicts of Interest: The author declares that he has no conflict of interest.

\section{References}

1. Watson, G.N. A Treatise on the Theory of Bessel Functions; Cambridge University Press: Cambridge, UK, 1962.

2. Abramowitz, M.; Stegun, I. Handbook of Mathematical Functions; U.S. National Bureau of Standards: Washington, DC, USA, 1964.

3. Baricz, Á. Some inequalities involving generalized Bessel functions. Math. Ineq. Appl. 2007, 10, 827-842. [CrossRef]

4. Baricz, Á. Generalized Bessel Functions of the First Kind. Ph.D. Thesis, Babes-Bolyai University, Cluj-Napoca, Romania, 2008.

5. Baricz, Á. Jordan-type inequalities for generalized Bessel functions. J. Inequal. Pure Appl. Math. 2008, 9, 39.

6. Baricz, Á.; Wu, S.H. Sharp Jordan-type inequalities for Bessel functions. Publ. Math. Debr. 2009, 74, $107-126$.

7. Baricz, Á.; Neuman, E. Inequalities involving generalized Bessel functions. J. Inequal. Pure Appl. Math. 2005, 6, 126. [CrossRef]

8. Baricz, Á. Functional inequalities involving Bessel and modified Bessel functions of the first kind. Expo. Math. 2008, 26, 279-293 [CrossRef]

9. Baricz, Á. Landen-type inequality for Bessel functions. Comput. Methods Funct. Theory 2005, 5, 373-379. [CrossRef]

10. Baricz, Á. Functional inequalities involving special functions. J. Math. Anal. Appl. 2006, 319, 450-459. [CrossRef]

11. Baricz, Á. Functional inequalities involving special functions II. J. Math. Anal. Appl. 2007, 327, 1202-1213. [CrossRef]

12. Toader, G. Some mean values related to the arithmetic-geometric mean. J. Math. Anal. Appl. 1998, 218, 358-368. [CrossRef]

13. Qi, F.; Shi, X.-T.; Liu, F.-F.; Yang, Z.-H. A double inequality for an integral mean in terms of the exponential and logarithmic means. Period Math. Hung. 2017, 75, 180-189. [CrossRef]

14. Lin, T.-P. The power mean and the logarithmic mean. Am. Math. Mon. 1974, 81, 879-883. [CrossRef]

15. Stolarsky, K.B. Generalizations of the Logarithmic Mean. Math. Mag. 1975, 48, 87-92. [CrossRef]

16. Páles, Z. Inequalities for differences of powers. J. Math. Anal. Appl. 1988, 131, 271-281. [CrossRef]

17. Alzer, H. Ungleichungen für Mittelwerte. Arch. Math. 1986, 47, 422-426. [CrossRef]

18. Yang, Z.-H.; Chu, Y.-M. On approximating the modified Bessel function of the first kind and Toader-Qi mean. J. Inequal. Appl. 2016, 2016, 40. [CrossRef]

19. Yang, Z.-H.; Chu, Y.-M.; Song, Y.-Q. Sharp bounds for Toader-Qi mean in terms of logarithmic and identric means. Math. Inequal. Appl. 2016, 19, 721-730. [CrossRef]

20. Yang, Z.-H.; Tian, J.-F.; Zhu, Y.-R. New sharp bounds for the modified Bessel function of the first kind and Toader-Qi mean. Mathematics 2020, 8, 901. [CrossRef]

21. Gould, H.W. Combinatorial Identities; Morgantown Printing and Binding: Morgantown, WV, USA, 1972. 